\title{
Hydroacoustic Sonification and Flow Pattern Investigation of Venous Pulsatile Tinnitus Using MEMS Hydrophone Sensing and Dye Flow Visualization Techniques: Pilot 3D Printing, Computational Fluid Dynamics, and Psychoacoustic Study
}

\author{
Yue-Lin Hsieh, ${ }^{1,2,3}$ Xing Wang, ${ }^{4}$ Xiaobing $\mathrm{Xu},{ }^{3,5}$ Yongzhen $\mathrm{Wu},{ }^{2,3}$ \\ Shenjiang Wang, ${ }^{5}$ Dengtao Yu, ${ }^{6}$ Yi-Chern Hsieh, ${ }^{7}$ and Wuqing Wang $2,3^{2}$ \\ ${ }^{1}$ Fudan University, No. 220 Handan Rd., Yangpu Dist., Shanghai 200433, China \\ ${ }^{2}$ Department of Otology and Skull Base Surgery, Eye, Ear, Nose, \& Throat Hospital, Fudan University, \\ No. 83 Fenyang Rd., Xuhui Dist., Shanghai 200031, China \\ ${ }^{3}$ NHC Key Laboratory of Hearing Medicine, No. 83 Fenyang Rd., Xuhui Dist., Shanghai 200031, China \\ ${ }^{4}$ Department of Acoustic Engineering, HI-KEY Technology Co., Ltd., \\ Xinjian East Rd., Minhang Dist., Shanghai 200235, China \\ ${ }^{5}$ Department of Radiology, Eye Ear Nose \& Throat Hospital, Fudan University, \\ No. 83 Fenyang Rd., Shanghai 200031, China \\ ${ }^{6}$ Institute of Gas Turbine, Shanghai Electric Gas Turbine Co., Ltd., \\ No. 333, Jiangchuan Rd., Minhang Dist., Shanghai 201100, China \\ ${ }^{7}$ Department of Power Mechanical Engineering, National Formosa University, \\ No. 64, Wunhua Rd., Huwei Township, Yunlin County 632, Taiwan
}

(Received July 1, 2021; accepted August 24, 2021)

Keywords: pulsatile tinnitus, hydrophone, computation fluid dynamics, dye flow visualization, 3D printing

Venous pulsatile tinnitus (PT) has been increasingly recognized in otology and neurotologic clinics. Although venous PT is surgically treatable, the surgical efficacy has unfortunately remained inconsistent owing to the relatively unknown mechanistic and psychophysical aspects of venous PT. We investigated the connection between the hydroacoustic and psychophysical characteristics of venous PT by integrating the outcomes attained from in vivo Doppler ultrasound, in vitro experiments, and psychoacoustic examination. The dye flow visualization technique used to investigate flow patterns was first introduced to this specific research topic. All 3D-printed and finite-element models were reconstructed on the basis of patients' magnetic resonance images. A MEMS hydrophone sensor was placed in the jugular bulb region intraluminally to study the operative alteration of the fluid-borne sound. Computational fluid dynamics (CFD) techniques were also implemented to cross-reference with the results of in vitro experiments. The in vitro and computational experimental outcomes showed that venous PT is the perception of intrasinus blood flow motion, in which the fluid flow amplitude may be associated with the ipsilateral flow volume and pressure gradient. In addition, the dye flow technique is beneficial for the study of intravascular flow patterns. However, in vitro/computational study results may potentially deviate from in vivo clinical measurements when the materials and

*Corresponding author: e-mail: wwuqing@eent.shmu.edu.cn

https://doi.org/10.18494/SAM.2021.3519

Revised October 8, 2021 
parameters used for experiments are varied. Thus, modifications and refinements of the applied sensing and materials are warranted in further experimental studies.

\section{Introduction}

The paired transverse-sigmoid sinuses are dural venous sinuses that serve as major conduits for venous drainage in the posterior cranial fossa. ${ }^{(1)}$ The cross-sectional area of the tortuous dural venous sinus can vary markedly, sometimes hypoplastically, when the unilateral transverse-sigmoid sinus is underdeveloped. ${ }^{(1)}$ Consequently, the bilateral sinus flow condition can vary significantly among the general population when juxtaposed. The positive intracranial pressure gradient drives the venous blood to return to the heart, whereby the condition that the motion of the blood flow becomes audible is known as venous pulsatile tinnitus (PT).(2)

Venous PT is an unusual, intrinsic, and most often unilateral perception of the dural venous sinus blood flow sound. ${ }^{(3)}$ The sensation of the flow sound is immediately alleviated or disappears when the ipsilateral jugular vein (outflow of the transverse-sigmoid sinus) is compressed. ${ }^{(4)}$ Furthermore, alterations of the intracranial pressure due to changes in the head and body position also affect the subjective loudness of PT. ${ }^{(5)}$ Although there has been no direct evidence indicating how the intrasinus flow becomes audible, clinical studies have suggested the progressive development of anatomical anomalies, i.e., sigmoid sinus wall anomalies (SSWA), and flow-based pathologies caused by transverse sinus stenosis (TSS) and/or idiopathic intracranial hypertension (IIH) as primary causes of PT generation. ${ }^{(6-8)}$ These anatomical and intracranial physiological anomalies have been increasingly documented by recent medical studies. $^{(2-9)}$

Equivalent to the perception of external sound stimuli or vibrations, venous PT is perceived by the inner ear (organ of Corti) situated in the temporal bone. The air- and/or bone-conduction sound transmission routes are major pathways for the intrasinus-generated flow sound to transmit to the inner ear. ${ }^{(3,9)}$ The former transmission route has been suggested as the major transmission pathway for PT, which is highly associated with a defective or thinned bony plate overlying the sigmoid sinus vessel wall. ${ }^{(7)}$ However, the bone-conduction transmission route has recently been indicated because low-frequency sound travels more efficiently through solid objects such as the temporal bone. ${ }^{(3,10)}$ Since the evaluation of the absolute amplitude of PT and the identification of transmission pathways of PT can be challenging, extrinsic sensing and computational techniques are crucial to understand the hydroacoustic characteristics of PT.

Sensing and material applications for the mechanistic investigation of PT are relatively novel. The rational and accurate acquisition of acoustic or vibration signals can be difficult in vivo. This renders in vitro experimental and computational fluid dynamics (CFD) studies necessary for mechanistic investigations, but only when the applied materials and/or the boundary conditions are reliably set. In real clinical settings, not only do the shape and flow characteristics in the transverse-sigmoid sinus vary vastly but also the human anatomical structures are exquisitely sophisticated. ${ }^{(3,10)}$ To that end, a uniformly given boundary condition can only provide a general indication for studies of the computational flow field. 
The intrasinus fluid-borne sound characteristics and the use of dye visualization showcasing the flow pattern have not yet been investigated. The major novelty and the primary aim of this study were to investigate the fluid-borne sound produced intravascularly using in vitro and computational methods. A MEMS hydrophone sensor was installed in patient-specific 3D-printed models to verify the computing accuracy of CFD results. The secondary aim of this study was to investigate the flow patterns of different vascular anomalies using a dye flow visualization technique. In addition, to update the CFD techniques used in our previous studies, we applied patient-specific flow volume/velocity data acquired from Doppler ultrasound. Knowledge of the connections among venous PT and its hydroacoustic and psychoacoustic characteristics can help clinicians optimize their treatment strategies.

\section{Materials and Methods}

\subsection{Clinical and radiologic data}

In this study, we recruited three patients with venous PT who had undergone extraluminal sigmoid sinus angioplasty from January to March 2021. The extraluminal compression surgical technique and related medical management were previously described. ${ }^{(3,5,11)}$ Their PT was successfully eliminated after surgery and did not recur during the three-month follow-up. Patient 1 had right-sided PT with ipsilateral sigmoid sinus wall dehiscence; patient 2 had leftsided PT with ipsilateral sigmoid sinus wall dehiscence; patient 3 had right-sided PT with ipsilesional sigmoid sinus diverticulum. Among the three patients, patient 3 had the most severe degree of TSS. All the patients were free of sensorineural hearing loss. Sigmoid sinus diverticulum and TSS are common vascular anomalies found in patients with venous PT. The former is the protrusion of the vascular wall into the mastoid cortex or air cells, forming an abnormal vascular out-pouch; the latter is a greater than $10 \%$ difference between the largest and smallest cross-sectional areas of the ipsilateral transverse sinus caliber. ${ }^{(3,7)}$ Sigmoid sinus wall dehiscence is the defect of a bony plate overlying the sigmoid sinus. ${ }^{(2)}$ A NUMARIS/4 workstation (SYNGO MRB17, Siemens AG) and Mimics 19.0 software (Materialise, Belgium) were used to analyze each preoperative and postoperative magnetic resonance angiogram/ venogram (MRA/V) and/or computed-tomography angiogram (CTA).

All the patients in this study provided written informed consent. Experimental measurements were conducted in accordance with the Declaration of Helsinki. Ethics approval was obtained from the Ethical Committee of the Eye, Ear, Nose, \& Throat Hospital in Shanghai, China.

\subsection{Applied materials and techniques for operative 3D-printed and computational models}

Patient-specific 3D finite-element and 3D-printed models were reconstructed on the basis of preoperative and postoperative MRA/V and/or CTA using Mimics 19.0 and 3-Matics 11.0 software (Materialise, Belgium). The 3D-printed patient-specific vascular models were produced by a 3D printer (Stratasys-J750, Israel) using the PolyJet 3D printing technique. A semitransparent 
Agilus30 rubber-like photopolymer was adopted. The tensile strength of the material was 2.4 $\mathrm{MPa}$, the tensile tear resistance was $5 \mathrm{~kg} / \mathrm{cm}$, and the Shore hardness was 30 Scale A. The overall thickness of the vascular wall was controlled from 0.9 to $1.1 \mathrm{~mm}$ and the slice height was $0.016 \mathrm{~mm}$. The lumen of the transverse sinus inlet and jugular outlet was elongated by 3 to $4 \mathrm{~cm}$ to smoothly connect with the customized connector. A Vernier caliper was deployed to mimic the surgical compression. The compression depth was measured from postoperative radiographic images as shown in Fig. 1.

For the computational models, the total tetrahedral grid number of all meshed computational models surpassed 300000. The flow inlet was set at the junction of the transverse sinus and the confluence of sinuses; the jugular outlet was established in the upper neck region. All sinus branches and intrasinus anatomical structures including arachnoid granulations and transverse sinus septa were removed in this study.

(a)
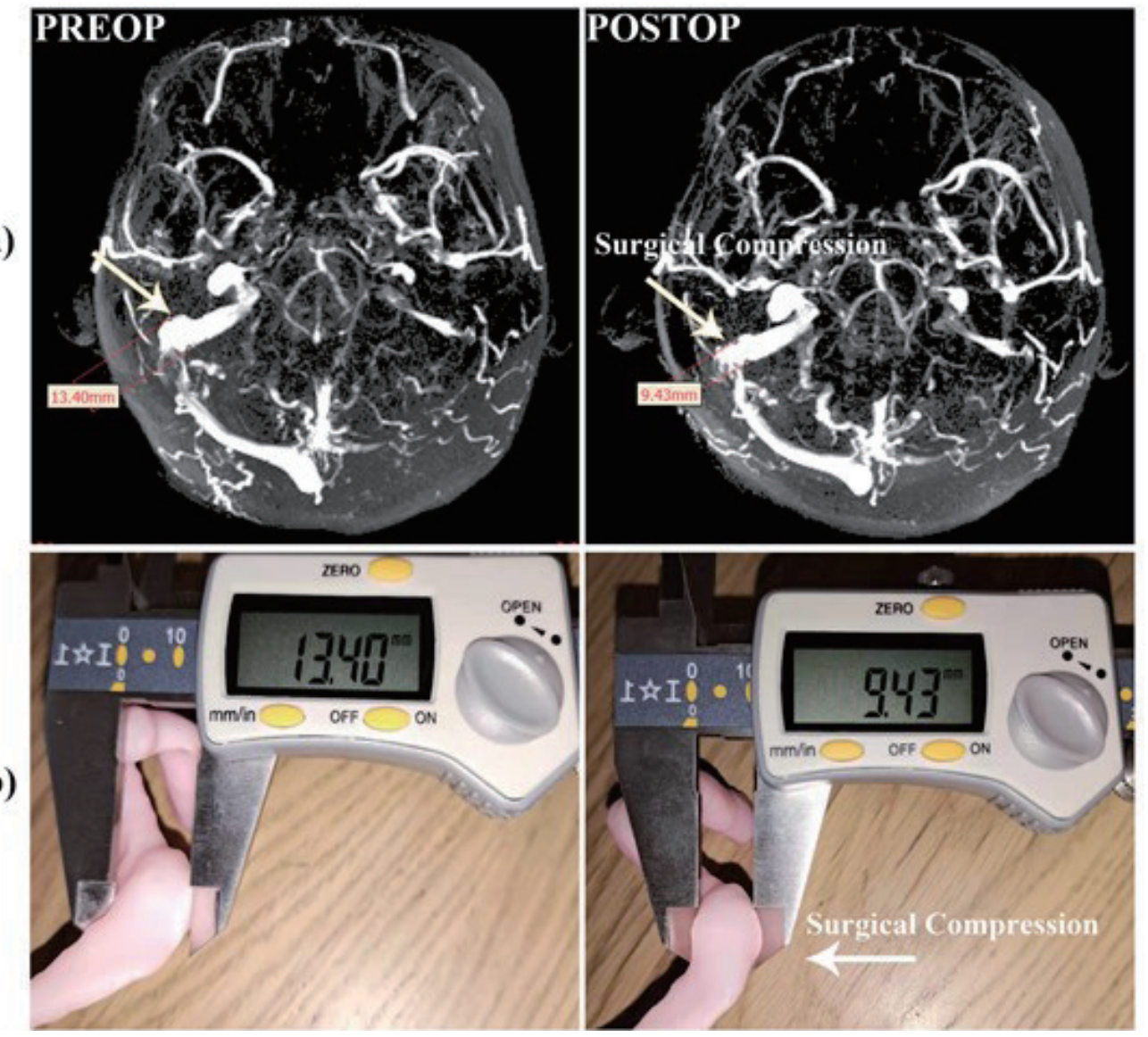

Fig. 1. (Color online) (a) 2D time-of-flight (TOF) magnetic resonance angiography (MRA)-transverse maximum intensity projection (MIP) of case 1 . The $29.6 \%$ operative compression of the transverse-sigmoid sinus junction diameter is shown. (b) Photographs showcasing the simulation of the operative compression using a Vernier caliper. 


\subsection{Establishment of flow circuit and dye visualization}

A 15-m-long silicon tube (inner/outer diameter $=9.6 / 14.6 \mathrm{~mm}$ ) used to establish the flow circuit was connected to a peristaltic AIPump600-KK25 pump $(6000 \mathrm{ml} / \mathrm{min})$ (Kamoer Fluid Tech Shanghai Co., Ltd., Shanghai, China) in a closed-loop fashion as shown in Fig. 2. The test models were fixed at the center of the water tank and submerged under liquid water. The output volume was adjusted on the basis of patient-specific flow data acquired from a color-coded Doppler ultrasound system (Esoate My Lab Class C, Italy). The measurement methods have been described in our previous works. To stabilize the in- and outflow of the flow field, customized plastic connectors were placed between the inlet/outlet and the silicon tubes. A hemostat was utilized to manually mimic the rhythmic motion of jugular/tricuspid valves in the jugular outlet region.

The fluorescent dye Rhodamine B with ethanol solution (red-orange color) was injected into the flow field of case 3 in the inlet region using a $50 \mathrm{ml}$ syringe and injection needle. A $365-\mathrm{nm}$ wavelength ultraviolet LED lamp (MicoTech TYNNOX ${ }^{\circledR}$, Shanghai, China) was placed above the transverse-sigmoid sinus junction to illuminate the flow field. To avoid vortex shedding, the needle was placed at the center of the lumen as parallel as possible to the direction of the working flow, and the injection speed was manually controlled to near the velocity of the flow field. The flow field was filmed using a portable camera with $240 \mathrm{fps} 1080 \mathrm{p}$ resolution. The motion and evolution of the illuminated fluid flow were post-analyzed using Tracker 5.15 software (Open Source Physics).

(a)

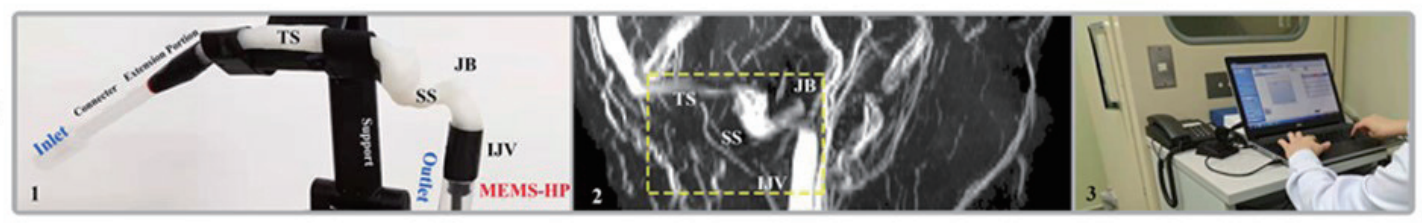

(b)
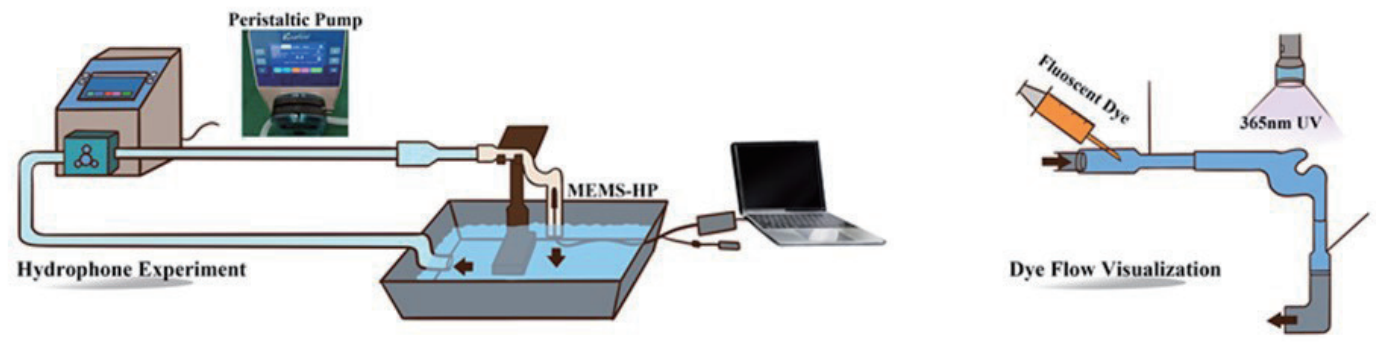

Fig. 2. (Color online) (a) (1) Display of the 3D-printed transverse-sigmoid sinus model of case 1. MEMS-HP indicates the MEMS hydrophone sensor; TS indicates transverse sinus; SS indicates sigmoid sinus; JB indicates jugular bulb; IJV indicates internal jugular vein. (2) 2D TOF magnetic resonance angiogram of case 1. (3) Photograph showcasing the psychoacoustic examination and soundproof testing environment. (b) Schematic diagram of the hydrophone experiment and dye flow visualization. 


\subsection{Installation of MEMS hydrophone sensor and data analysis}

A $7 \times 15 \mathrm{~mm}$ customized MEMS vector hydrophone sensor (STQ20-20000, Anbuleila Automation Technology Co., Ltd., Hangzhou, China) with a built-in $40 \mathrm{~dB}$ amplifier was inserted endoluminally in the jugular bulb region to detect the generated intraluminal fluidborne sound as shown in Fig. 2. Owing to the shape and the size of the hydrophone sensor, detection above the jugular bulb was impossible in all 3D-printed models. The measurement of case 3 was excluded due to the small cross-sectional area at the internal jugular vein. The sound pressure sensitivity of the hydrophone was $-180 \mathrm{~dB}$ re $1 \mathrm{~V} / \mu \mathrm{Pa}$. The sensitivity of the frequency bandwidth was $20 \mathrm{~Hz}-20 \mathrm{kHz}$.

The signal data (audible volume) recorded by the hydrophone was converted to the estimated sound pressure level (SPL) using the following equations:

$$
\begin{gathered}
S P L+M=20 \log _{10}\left(\frac{U}{1 \mu \mathrm{Pa}}\right), \\
S P L+M=20 \log _{10}\left(\frac{U}{20 \mu \mathrm{Pa}}\right),
\end{gathered}
$$

where $M$ is the transducer sensitivity $(-180 \mathrm{~dB}$ re $1 \mathrm{~V} / \mu \mathrm{Pa})$ and $U$ is the sound pressure.

In water, the common reference is $1 \mu \mathrm{Pa} ; 20 \mu \mathrm{Pa}$ is the hearing-related reference pressure in air. These two references are therefore $26 \mathrm{~dB}$ apart $(20 \log 20=26)$. Hence, the expressions using the estimated SPL are reformulated as

$$
\begin{aligned}
& S P L_{\text {water }}=20 \log _{10} U+100, \\
& S P L_{\text {air }}=20 \log _{10} U+126 .
\end{aligned}
$$

The post-analysis of the recorded audio data was conducted in line with our previous studies. ${ }^{(3,12)}$ The spectrotemporal analysis and conversion of the decibel scale were carried out using the short-time Fourier transform and an analog A-weighting filter using MATLAB R2017a.

\subsection{Boundary conditions and modified hydroacoustic flow field simulation based on patient-specific Doppler ultrasound}

For the simulation of the flow field, the velocity inlet of each model was set according to the patient-specific velocity data acquired from the Doppler ultrasound. Fluent (ANSYS, Inc. Cecil Township, PA, USA) and Actran 17.2 (MSC, Free Field Technology, Belgium) software were deployed to calculate the flow and acoustic fields. The transient inlet velocity was set according to the patient-specific Doppler data, and the large-eddy simulation model was used for the 
calculation of the flow field. The boundary conditions of the moving vascular wall were set as follows: Young's modulus, 1.26 MPa; Poisson's ratio, 0.3; density, $1.05 \mathrm{~g} / \mathrm{cm}^{3}$. ${ }^{(13)}$ The vascular wall thickness was set as $2 \mathrm{~mm}$. The density of Newtonian incompressible blood was $1050 \mathrm{~kg} / \mathrm{m}^{3}$, and the dynamic viscosity was $0.00345 \mathrm{~Pa} \cdot \mathrm{s}$. As the Lighthill and Möhring [Eq. (7)] equations are derived from the Navier-Stokes equations [Eqs. (5) and (6)], the flow fluctuations and their associated length scales are present in the solution.

$$
\begin{gathered}
\nabla \cdot \boldsymbol{u}=0 \\
\rho \frac{\partial \boldsymbol{u}}{\partial t}+\rho \boldsymbol{u} \cdot \nabla \boldsymbol{u}=-\nabla \boldsymbol{p}+\nabla \cdot \boldsymbol{\tau}+\rho \boldsymbol{g} \\
4 \pi c_{0}^{2} \rho^{\prime}(x, t)=\frac{\partial^{2}}{\partial x_{i} \partial x_{j}} \iiint_{V} \frac{T_{i j}}{|x-y|}
\end{gathered}
$$

In the analysis, we filtered the hydrodynamic part of the pressure field using the following acoustic perturbation equation proposed by Ewert et al.:(14)

$$
\frac{\partial^{2} p}{\partial t^{2}}-c^{2} \frac{\partial^{2} p}{\partial x_{i} \partial x_{i}}=-\frac{\partial^{2} \phi_{3}}{\partial t^{2}}
$$

where $\phi_{3}$ is calculated by solving

$$
\frac{\partial^{2} \phi_{3}}{\partial x_{i} \partial x_{i}}=\frac{\partial^{2} \rho v_{i} v_{j}}{\partial x_{i} \partial x_{j}}
$$

The simulated acoustic data were exported and subjected to spectrotemporal analysis analogous to the methodologies described above. Owing to the complexity of the temporal bone structures, the sound impedance and fluid-structure vibration between the temporal bone and vascular wall were not included. At least four circulatory intrasinus flow cycles were calculated.

\subsection{Psychoacoustic testing of PT frequency and loudness}

Audiometric examinations were performed in a soundproof booth using a Madsen Astera audiometer (Natus Medical Denmark ApS, Taastrup, Denmark) and TDH-39P headphones (Telephonics Ltd., Farmingdale, NY) as shown in Fig. 2. The purpose of performing psychoacoustic testing was to evaluate the subjective loudness and frequency of PT perceived by the participants. Prior to the psychoacoustic testing, the participants were carefully instructed to discern the difference between "loudness" and "pitch" and to neglect the rhythmic components of both PT and given stimuli. During frequency matching, pure-tone stimuli and $1 / 3$ octave narrow-band noise at each (center) frequency of 125, 250,500, and $750 \mathrm{~Hz}$ and 1, 1.5, 2, 3, 4, 6, 
and $8 \mathrm{kHz}$ were presented from the audiometer. Narrow-band noise was given from $125 \mathrm{~Hz}$ upwards to $8 \mathrm{kHz}$. Once the bandwidth was confirmed, participants were then instructed to compare the given narrow-band noise downwards. The subjects were informed to raise their hand and choose the given stimulus that best matched the pitch of their PT. All sound stimuli were given unilaterally to the contralesional ear through headphones. During the loudness matching, the amplitude of each stimulus was increased from the hearing threshold level by $1 \mathrm{~dB}$ steps, and during each step, the patient was asked if the loudness of the given sound matched her PT. Finally, participants were asked to rate the likeness of the pitch matching using a numerical rating scale (0 to 100$)$.

\section{Results}

\subsection{Operative alterations of bilateral outflow volume measured by Doppler ultrasound}

Table 1 shows the operative alterations of the bilateral jugular outflow hemodynamics. From the viewpoint of total bilateral outflow volume, the postoperative bilateral flow volume increased in cases 2 and 3. Case 1 had a slight reduction of total bilateral outflow volume. By juxtaposing the ipsilesional outflow volume, the outflow volumes of cases 1 and 2 were reduced by 22.5 and $46.3 \%$, respectively. Interestingly, the ipsilesional outflow volume increased by $49.2 \%$ in case 3. There was an evident switch of the outflow volume on both sides after extraluminal compression surgery in case 2 . The pulsatility index varied among participants and was reduced bilaterally in all postoperative cases.

\subsection{Results of MEMS hydrophone sensor detection and CFD hydroacoustic simulation}

For the hydrophone experiment, Fig. 3 and Table 2 both demonstrated that cases 1 and 2 had significant reduction in the peak amplitude of the fluid-borne sound detected by the hydrophone sensor. The largest estimated preoperative flow amplitude of case 1 within the humanly audible frequency range $(20 \mathrm{~Hz}-20 \mathrm{kHz})$ was $103.5 \mathrm{~dB}$ SPL at $98.5 \mathrm{~Hz}$, which was reduced to $100.4 \mathrm{~dB}$ SPL at $20.5 \mathrm{~Hz}$ after the volumetric change and the extraluminal compression of the

Table 1

Operative and Doppler ultrasonographic data of the three cases.

\begin{tabular}{|c|c|c|c|c|c|c|}
\hline & \multirow{2}{*}{$\begin{array}{c}\text { Degree of } \\
\text { extraluminal } \\
\text { compression (\%) }\end{array}$} & \multirow[t]{2}{*}{ Preop/Postop } & $\begin{array}{c}\text { Flow volume } \\
(\mathrm{g} / \mathrm{s})\end{array}$ & $\begin{array}{c}\text { Peak flow } \\
\text { velocity }(\mathrm{m} / \mathrm{s})\end{array}$ & $\begin{array}{c}\text { Mean flow } \\
\text { velocity }(\mathrm{m} / \mathrm{s})\end{array}$ & $\begin{array}{l}\text { Pulsatility } \\
\text { index }\end{array}$ \\
\hline & & & \multicolumn{4}{|c|}{ Ipsilesional/Contralesional } \\
\hline \multirow{2}{*}{ Case 1} & \multirow{2}{*}{29.6} & Preop & $32.9 / 10.4$ & $0.604 / 0.619$ & $0.413 / 0.39$ & $0.92 / 2.44$ \\
\hline & & Postop & $25.5 / 7.19$ & $0.46 / 0.395$ & $0.32 / 0.27$ & $0.65 / 1.87$ \\
\hline \multirow{2}{*}{ Case 2} & \multirow{2}{*}{59.4} & Preop & $17.9 / 9.8$ & $0.5 / 0.359$ & $0.312 / 0.182$ & $1.23 / 1.34$ \\
\hline & & Postop & $9.6 / 20.8$ & $0.211 / 0.417$ & $0.167 / 0.382$ & $0.17 / 0.12$ \\
\hline \multirow{2}{*}{ Case 3} & \multirow{2}{*}{69.5} & Preop & $5.2 / 3.28$ & $0.3 / 0.3$ & $0.214 / 0.21$ & $0.5 / 0.81$ \\
\hline & & Postop & $10.25 / 2.3$ & $0.579 / 0.167$ & $0.414 / 0.127$ & $0.25 / 0.22$ \\
\hline
\end{tabular}



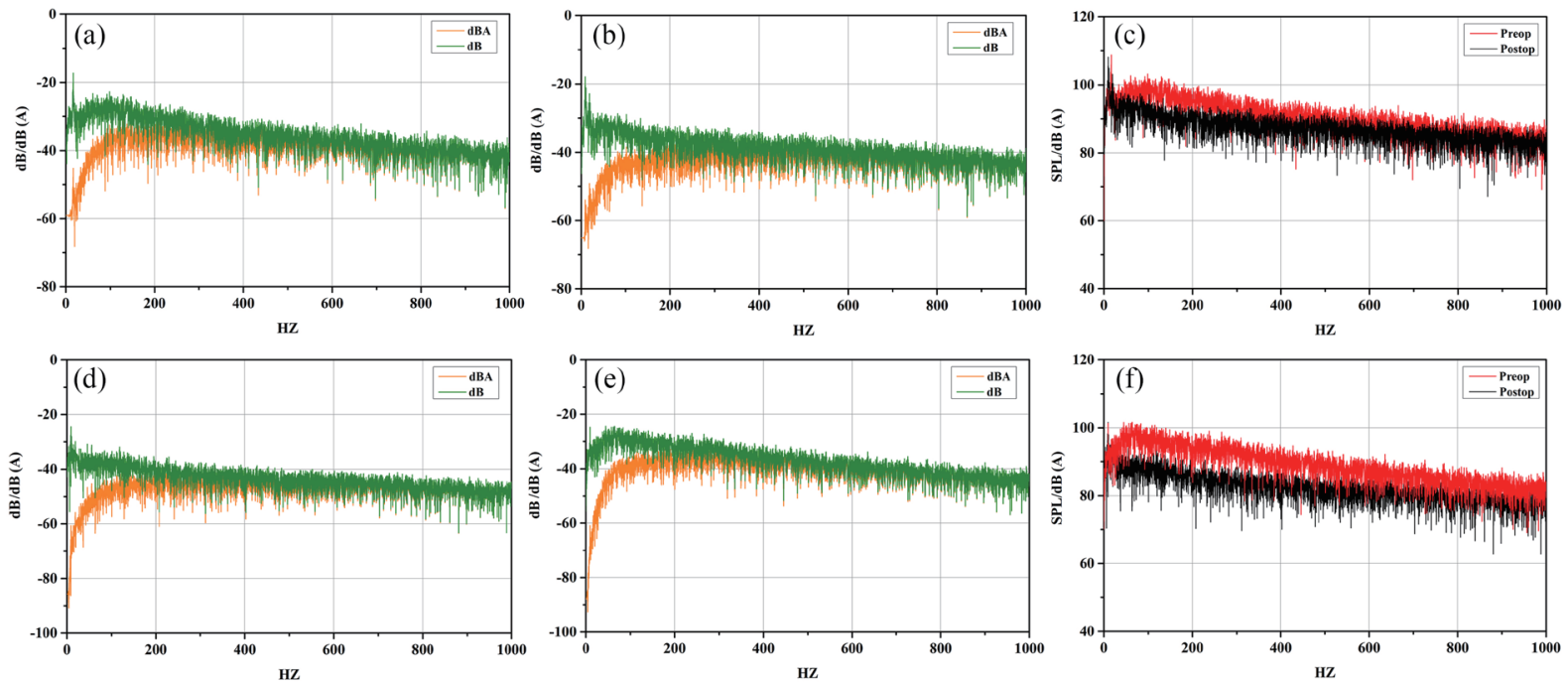

Fig. 3. (Color online) Results of the fluid-borne sound detected by the MEMS hydrophone sensor placed in the jugular bulb region of the 3D-printed models. (a) Preoperative state of case 1. (b) Postoperative state of case 1. (c) Operative alteration of the amplitude of the fluid-borne sound of case 1 (reference pressure in air, $P_{\text {ref }}=20 \mu \mathrm{Pa}$ ). (d) Preoperative state of case 2. (e) Postoperative state of case 2. (f) Operative alteration of the amplitude of the fluidborne sound of case 2 (reference pressure in air, $P_{\text {ref }}=20 \mu \mathrm{Pa}$ ).

Table 2

Hydroacoustic and hemodynamic characteristics of the patients measured using MEMS hydrophone sensor and CFD.

\begin{tabular}{llcccc}
\hline & \multicolumn{2}{c}{ MEMS hydrophone/CFD hydroacoustics } & \multicolumn{2}{c}{ CFD hemodynamics } \\
\hline \multirow{2}{*}{ Case 1 } & $\begin{array}{c}\text { Peak amplitude } \\
\left(\text { Hydrophone } / \text { CFD }^{*}\right)(\mathrm{dB})\end{array}$ & $\begin{array}{c}\text { Frequency at peak amplitude } \\
(\text { Hydrophone/CFD) }(\mathrm{Hz})\end{array}$ & $\begin{array}{c}\text { Peak velocity } \\
(\mathrm{m} / \mathrm{s})\end{array}$ & $\begin{array}{c}\text { Pressure } \\
\text { gradient }^{\#}\left(\mathrm{~N} / \mathrm{m}^{3}\right)\end{array}$ \\
& Postop & $103.5 / 108.6$ & $98.5 / 47.5$ & 0.73 & 15.2 \\
\multirow{2}{*}{ Case 2 } & Preop & $100.4 / 91.4$ & $20.5 / 51.4$ & 0.58 & 21.9 \\
& Postop & $95.6 / 122.2$ & $46.5 / 58.9$ & 0.67 & 32.4 \\
\hline
\end{tabular}

* indicates the hearing-related reference pressure in air in the unit of $\mathrm{dB}$ SPL $(\mathrm{ref}=20 \mu \mathrm{Pa})$

\# indicates the pressure gradient from the inlet to the outlet of the transverse-sigmoid sinus.

transverse-sigmoid junction. In case 2, the largest preoperative flow amplitude was $101.6 \mathrm{~dB}$ SPL at $46.5 \mathrm{~Hz}$, whereas the postoperative peak flow amplitude was 95.1 dB SPL at $63.7 \mathrm{~Hz}$.

In CFD hydroacoustic simulations, the computed peak amplitude of the intrasinus flow was 108.6 dB SPL at $47.5 \mathrm{~Hz}$ in case 1, whereas the postoperative peak amplitude was $91.4 \mathrm{~dB}$ SPL at $51.4 \mathrm{~Hz}$. In case 2, the preoperative peak amplitude was $122.2 \mathrm{~dB}$ SPL at $58.9 \mathrm{~Hz}$; the postoperative peak amplitude was $118.6 \mathrm{~dB}$ SPL at $42.3 \mathrm{~Hz}$. The operative amplitude map pressure and flow visualization of the entire ipsilesional transverse-sigmoid sinus and jugular bulb region of case 1 are shown in Fig. 4. All hemodynamic CFD results are shown in Table 2. 


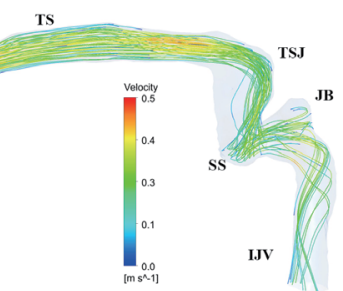

(a)
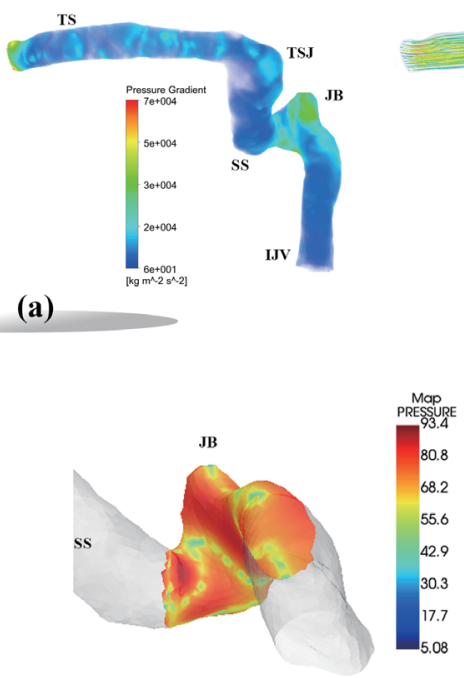

(c)

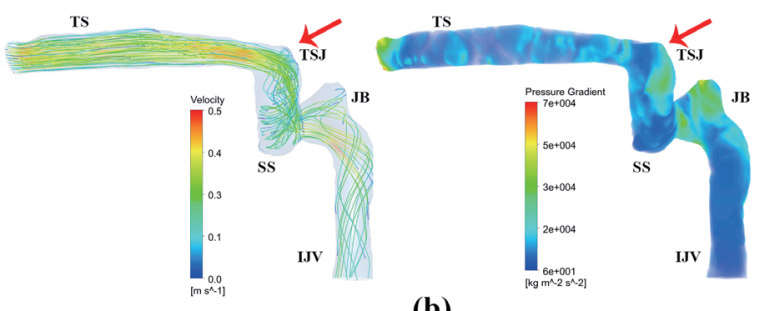

(b)

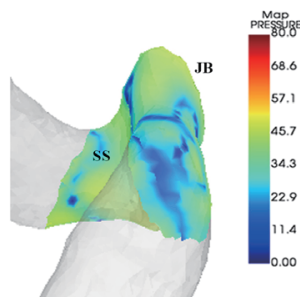

(d)

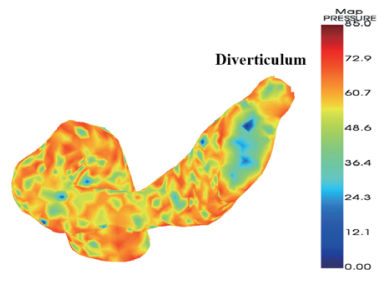

(e)
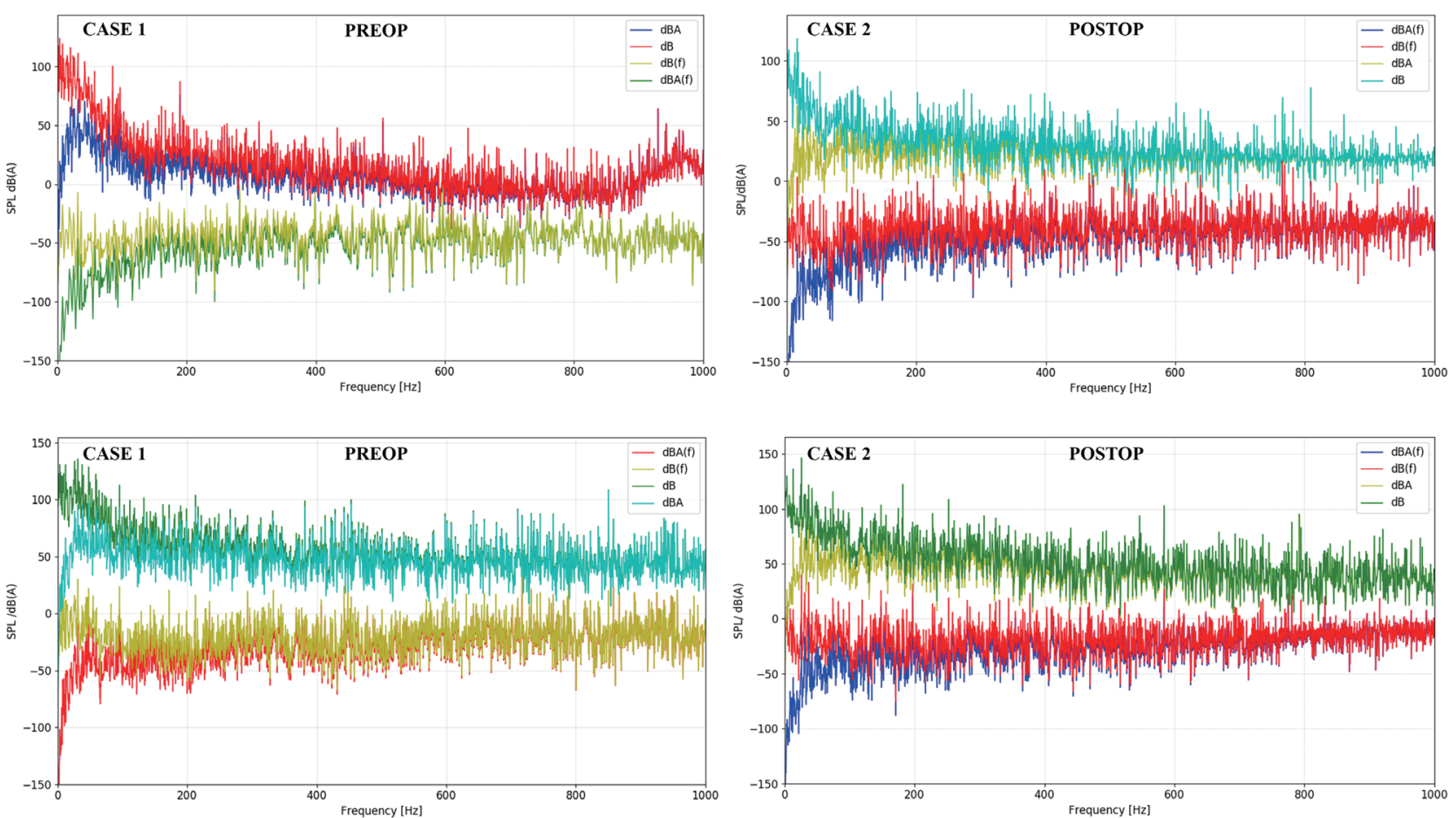

Fig. 4. (Color online) Flow and acoustic fields of the ipsilesional transverse-sigmoid sinus. The lower panel displays the operative acoustic profiles (amplitude spectra) of cases 1 and 2. Note that the filtered (f), unfiltered, dB SPL, and dBA data are simultaneously displayed. (a) Preoperative hemodynamic state of case 1, showing flow field velocity and streamline (left panel) and pressure gradient (right panel). (b) Postoperative hemodynamic state of case 1, showing flow field velocity and streamline (left panel) and pressure gradient (right panel). (c) Preoperative acoustic field at the jugular bulb of case 1. (d) Postoperative acoustic field at the jugular bulb of case 1. (e) Crosssectional view of the acoustic field of the diverticulum and transverse-sigmoid junction in case 3 . 


\subsection{Flow patterns using dye flow visualization}

Results of the dye flow visualization are shown in Fig. 5. The estimated mean velocity of the illuminated working flow was $0.208 \mathrm{~m} / \mathrm{s}$. Initially, the flow velocity increased as the flow passed through the TSS. A jetlike flow formed after the flow passed through the TSS, where its transient velocity was estimated to surpass $0.75 \mathrm{~m} / \mathrm{s}$. The transverse-sigmoid junction and the jugular bulb were the regions where the flow velocity markedly decreased. This was exhibited by the increase in the visible rotational flow motion, which caused flow stagnation to develop from the jet flow that impacted on the transverse-sigmoid junction. Note that the illuminated flow first entered the upper curve of the sigmoid sinus, possibly due to the low pressure gradient, which preceded the flow of the dye entering the diverticulum. This indicated that the sudden expansion of the sinus lumen, i.e., the diverticulum or enlargement transverse-sigmoid junction, and the abrupt changes in the flow direction led to the increase in the regional pressure gradient. Such a physical phenomenon was likewise seen in the jugular bulb region, where the velocity of
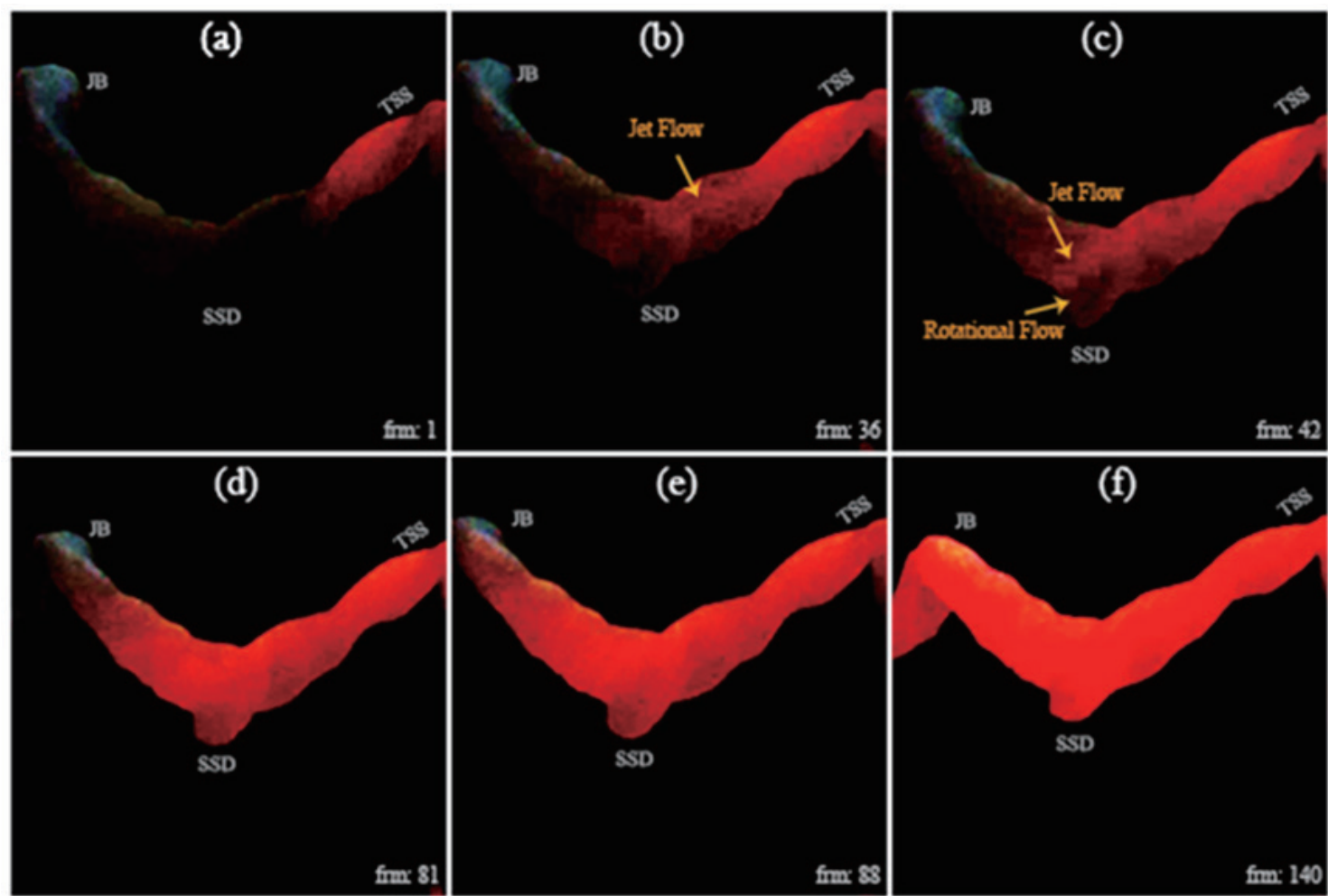

Fig. 5. (Color online) Dye flow visualization of the flow field of case 3 with sigmoid sinus diverticulum (SSD); frm indicates frame number. (a) Illuminated flow passing through a TSS. (b) Formation of the post-stenosis jet flow. (c) Occurrence of rotational flow after jet flow impacting on the transverse-sigmoid junction vascular wall and possible visualization of flow separation. (d) Full dye illumination filling the SSD. (e) Flow stagnation in jugular bulb region. (f) Illumination of the entire transverse-sigmoid sinus lumen. 
the dye flow entering the jugular bulb significantly decreased. The jugular bulb exit velocity once again increased significantly due to the low pressure gradient, demonstrating that the flow conditions in the sigmoid sinus were highly chaotic and segmental.

\subsection{Results of psychoacoustic testing and spectrotemporal analysis}

Results of the psychoacoustic matching and likeness rating are shown in Table 3. The loudness values of PT were 23 and $14 \mathrm{dBSL}$ in cases 1 and 2, respectively. The frequency matching demonstrated that the subjective harmonic range of PT extended over the $1 / 3$ octave narrow-band noise with a center frequency of $500 \mathrm{~Hz}$. Interestingly, both participants chose the $1 / 3$ octave narrow-band noise with the center frequency of 125 or $250 \mathrm{~Hz}$ that best matched their PT frequency. In contrast to the hydrophone-measured/CFD-simulated audio data, the likeness of the Doppler ultrasound recordings of both participants was rated the highest. The harmonic range of both hydrophone and CFD results was comparatively low (see Fig. 6 for the spectrotemporal analysis of the audio data). In addition, the disparity between the manually controlled and in vivo rhythmic components of PT was also an influencing factor that caused the low rating of likeness for the hydrophone recordings.

\section{Discussion}

\subsection{Combining in vitro experimental outcomes and in vivo detection of the fluid-borne sound}

This is the first study to use a MEMS hydrophone sensor to capture the fluid-borne sound generated by the working flow inside 3D-printed transverse-sigmoid sinus models. It is clear that venous $\mathrm{PT}$ is the perception of intrasinus blood flow motion. The blood flow sound of venous PT was previously studied using 3D-printed models via different recording methods. ${ }^{(13,15)}$ In congruence with a recent in vitro study conducted by Valluru et al. that found that the main power of PT fell below $600 \mathrm{~Hz}$ using an electrical stethoscope, ${ }^{(15)}$ both our in vivo ultrasonography and in vitro experiments showed similar results regarding frequency components. However, since the properties of the materials used for 3D-printed models vary significantly among the studies, to avoid sound impedance and sound transmission loss caused by the applied materials, an intravascularly placed hydrophone allows the analysis of the magnitude of the flow amplitude generated inside the sigmoid sinus. Our in vitro data

Table 3

Results of psychoacoustic testing of the participants.

\begin{tabular}{lccc}
\hline & Loudness (dBSL) & Frequency $^{*}(\mathrm{~Hz})$ & $\begin{array}{c}\text { Subjective Likeness Rating } \\
\text { (Doppler/Hydrophone/CFD) }\end{array}$ \\
\hline Case 1 & 23 & $125 / 250$ & $90 / 70 / 80$ \\
Case 2 & 14 & $125 / 250 / 500$ & $85 / 60 / 65$ \\
\hline
\end{tabular}

${ }^{*}$ The frequency matching was performed using $1 / 3$ octave narrow-band noise with center frequency of 125/250/500/750/1000 Hz. 
(a)

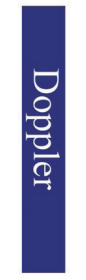

(b)

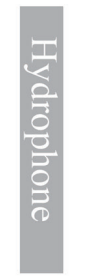

(c)

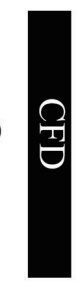

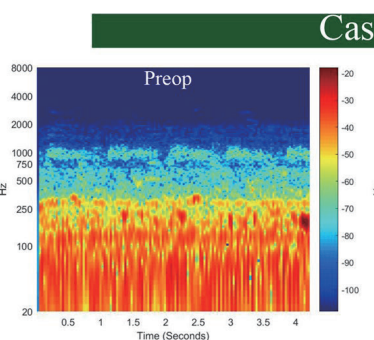
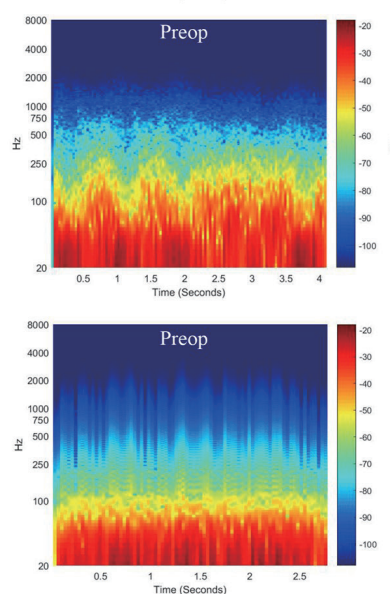

\section{Case 1}
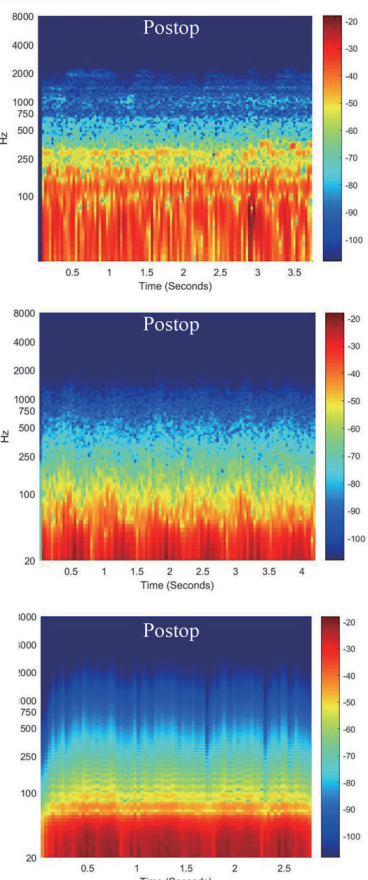
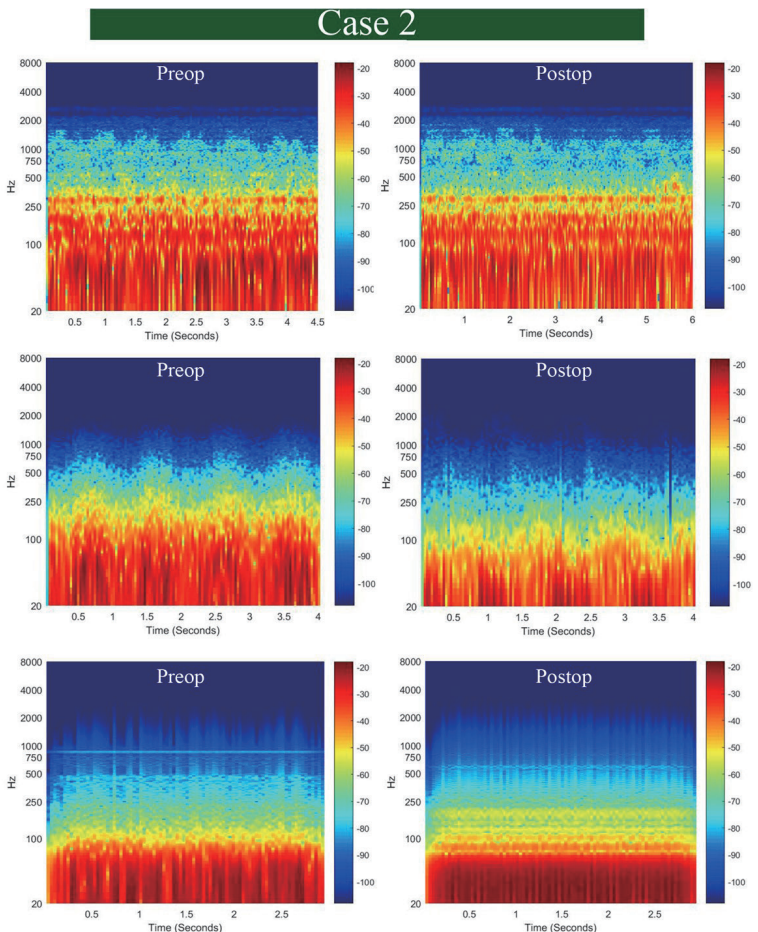

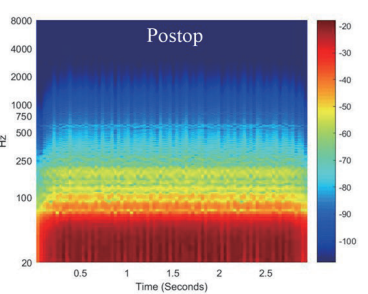

Fig. 6. (Color online) Results of spectrotemporal analysis of the preoperative and postoperative audio profiles acquired from (a) Doppler ultrasound, (b) hydrophone sensor, and (c) CFD simulation.

demonstrated that the estimated magnitude of the intraluminal flow amplitude can surpass 100 $\mathrm{dB}$ SPL. Because the fluid and air pressure waves concomitantly contribute to the acoustic production, it is plausible, according to our clinical data, that the perceived loudness of PT should be more strongly related to the filtered acoustic results (air pressure data alone). Therefore, the interference of the fluid pressure wave must be excluded to extract desirable computational results. Another interesting computational finding is that the amplitude of the sinus flow was larger near the vascular surface than at the luminal center. It is likely that the higher fluid shear stress and vorticity may contribute to sound generation. Hence, the acquisition of amplitude data can vary markedly depending on the location where sound data are collected.

The current clinical outcomes suggest that longitudinal changes in the flow volume affect the loudness of PT. This means that the reduction of the generated flow amplitude is, in part, related to the decrease in ipsilesional flow volume, which can be associated with surgical reshaping of the transverse-sigmoid junction. As in case 3 , whose PT disappeared without evident alteration of the ipsilesional flow volume, it is likely that the reconstruction of the sound-insulating sigmoid sinus bony plate prevented the sound transmission of PT to the inner ear, or the PT is eliminated simply because the intrasinus vortical structures are surgically eradicated. ${ }^{(3)}$ However, the difference among fluid-borne sounds produced by vortical structures needs to be further investigated. Furthermore, merely reshaping the vascular silhouette without deliberately isolating the PT transmission route, or vice versa, may not suffice to decrease the PT amplitude. ${ }^{(3)}$ 
Hence, reduction of the flow amplitude via addressing vascular anomalies and reconstruction of the defective sigmoid sinus bony plate using sound-insulating materials are both indicative for transtemporal surgeries.

\subsection{Transmission of flow sound}

There has been no evidence indicating whether venous PT develops alongside the alteration of intrasinus flow characteristics. Venous PT has been clinically theorized to transmit via airand bone-conduction pathways. ${ }^{(3,9,10)}$ According to our previous in vivo measurements using displacement sensors, the motion of the sigmoid sinus vessel wall seems unlikely the major cause of PT. ${ }^{(10)}$ This raises a postulation that the airtight condition of the mastoid cavity and/or the defective bony plate may facilitate the fluid-borne sound transmission to the inner ear. In addition to the fluid-borne sound alone, the hydrodynamic pressure may also contribute to PT sound transmission, typically via the bone-conduction route. For some patients, PT persists after mastoidectomy under local anesthesia, although the PT of a few patients disappears once the mastoid is cut open. ${ }^{(3,8,10)}$ This clearly indicates that the airtight environment plays an important role in the transmission of PT, and when the mastoid air cells are eliminated, it is likely that the flow sound energy dissipates to the open air, rendering PT inaudible. A positive response to the water occlusion test or the ear-cover test (adding pressure to the middle ear) may suggest the compromised integrity of the sigmoid sinus bony plate for patients with SSWA. ${ }^{(10,16)}$ It was also found that over $1 \mathrm{~g}$ of external force can preclude the sound energy transmitting to the middle ear. For those whose PT persists after mastoidectomy, it is likely that the bone-conduction route plays the predominant role in PT transmission. This phenomenon is more common than the PT disappearing during surgery, indicating that the bone-conduction route may also be a contributing factor causing PT transmission to the inner ear.

\subsection{Intrasinus and intradiverticular flow patterns}

This is the first study to use the dye flow visualization technique to investigate the intrasinus flow pattern. The intrasinus flow pattern was previously revealed using CFD methods or in vivo radiological modalities. However, the acquisition of flow data using these techniques may be limited to the applied boundary conditions or parameters. The current dye flow visualization technique was able to highlight the prominent features of the working fluid flow. In line with previous CFD/radiologic studies, the intrasinus flow velocity fluctuates among different segments. ${ }^{(3,5)}$ It is also evidenced that the vortical flow structures originate from the irregular shape of the transverse-sigmoid junction, where a distal TSS, if present, may potentially increase the regional flow velocity and vorticity. Swirling and rotational flows also appear in the jugular bulb region, indicating that sound production at the distal portion of the transverse-sigmoid sinus, typically where dehiscence can occasionally be found, should not be ruled out. By combining the dye flow visualization and CFD results, the transverse-sigmoid junction and jugular bulb flow regions have a lower flow velocity, possibly because of the resultant highpressure gradient due to the fluctuating cross-sectional luminal area. 


\subsection{Psychoacoustic aspect of PT}

The frequency bandwidth of PT is generally below $1.5 \mathrm{kHz}$, occasionally extending up to $1.5-2 \mathrm{kHz} .{ }^{(3,17)}$ This, in turn, can be verified by psychoacoustic testing, during which patients generally indicate high resemblance of their PT sound to the given 1/3 octave narrow-band noises with center frequency of 125/250/500/750 Hz. Song et al. first analyzed patient PT of different vascular origins captured by the trans-external auditory canal recording technique using spectrotemporal analysis. ${ }^{(18)}$ They indicated that the acoustic characteristics of PT may potentially vary among different vascular etiologies. As shown by the present study, both hydrophone sensing and CFD were able to capture the low-frequency fluid-borne sound similar to the in vivo blood flow sound measured by Doppler ultrasound. The frequency of venous PT can also be obliquely exhibited by some patients presenting ipsilateral low-frequency sensorineural hearing loss, typically below $500 \mathrm{~Hz}$, although such a clinical presentation likely results from the tinnitus masking effect (pseudo-hearing loss). ${ }^{(16)}$ Regarding the loudness perception of venous PT, our previous audiometric study found that the mean loudness of the 55 tested participants was approximately $17 \mathrm{dBSL} .{ }^{(19)}$ There are two influencing factors that may explain the significantly lowered perceived loudness of PT: 1) the power of the blood flow sound is greatly reduced during its transmission to the inner ear, and 2) sound inhibition is regulated by the central nervous system. PT is occasionally inaudible by surgeons when the mastoid is cut open, and further study on PT transmission within the aerial and airtight middle ear cavity needs to be conducted. In addition, subjective outcomes may also play a role in the perception of PT loudness, as anxiety, depression, and sleep disorders prevail in the demographics of people with venous PT. ${ }^{(19)}$

\subsection{Study limitations}

This study has several limitations. Firstly, the small study sample may hinder a comprehensive understanding of the correlation between the hydroacoustic characteristics and flow pattern, although our current data have shown that the generated flow sound amplitude was predominantly determined by the ipsilateral flow energy and flow patency. As the sound transmission loss and sound transmission pathways in the mastoid bone were excluded from this study, further investigation of this specific topic will be carried out. Secondly, the insertion of the MEMS hydrophone sensor at the transverse-sigmoid junction was nearly impossible in all anatomical models, a problem that can only be solved if a smaller hydrophone sensor is manufactured. It was also not possible to differentiate the generated flow sound close to or away from the near-wall regions. Thirdly, it is difficult for the materials used in this study to substitute for human anatomical structures. To our knowledge, the Shore hardness and tensile tear resistance of the current applied material are the closest to those of the dural venous sinus wall, but the tactile impression, the wall thickness, and its characteristics still remain considerably different from those of the human vessel wall. Another important point is that manually mimicking the action of heart systole and diastole can be physiologically imprecise. This is because the rhythmic pattern of the current in vitro simulated flows partly deviated from the 
acquired Doppler velocity spectrum. Fourthly, a 1/3 octave narrow-band noise without pulsing does not perfectly represent PT. However, most test subjects will indicate the given noise with a high resemblance to PT in their first testing experience. Therefore, we consider this a rational method to test the loudness of PT. Lastly, the 3D-printed models were semitransparent, and the flow material was water, not blood, which should be modified in future studies. Henceforth, the intrasinus vortical structures displayed using the dye flow visualization may not be sufficiently vibrant.

\section{Conclusions}

In this study, we updated the technical aspects of both sensing and computation techniques reported in previous medical-based studies to investigate the generation of the intrasinus fluidborne sound. Conflating in vitro experimental outcomes with in vivo measurements can provide valuable indications for the future development of surgical techniques and the improvement of surgical efficacy. The connections found among venous PT, hydroacoustic, and psychoacoustic characteristics are concluded as the following clinical and experimental implications:

1) Clinical implications:

- As indicated by the in vivo Doppler measurements, redistribution of the total sinus outflow volume can occur after extraluminal compression surgery that specifically targets the dural venous sinus hemodynamics. The medical and pathophysiological aspects of this issue should be further assessed.

- The reduction of postoperative flow amplitude may not be evident if the variation of the intrasinus flow volume and flow pressure gradient is insignificant, which indicates that isolating the sound transmission of PT via sigmoid sinus plate dehiscence into the inner ear may be indicative of the surgical goal.

2) Experimental implications:

- Acquiring both patient-specific pre- and postoperative velocity data is one of the utmost crucial prerequisites for the investigation of hydroacoustic characteristics in the transversesigmoid sinus flow domain.

- Audio data both acquired from the MEMS hydrophone sensor and simulated by the CFD technique indicate that venous PT is the perception of fluid motion. The acoustic profile of venous PT varies, which is associated with the ipsilateral flow volume, flow velocity, and flow pressure gradient.

- Different materials and parameters applied to in vitro 3D-printing experiments and/or CFD simulations may greatly alter the outcome of interest. Thus, continuous refinements of experiment methodologies and the use of patient-specific flow data are encouraged in future investigative studies.

- The dye flow visualization technique can provide transient and detailed flow visualization of the prominent intrasinus flow pattern, typically for flow models that present TSS and SSD. Further enhancements of dye flow visualization techniques and the transparency of flow models are warranted. 


\section{Acknowledgments}

WW was supported by the National Natural Science Foundation of China (no. 81670933) and the Natural Science Foundation of Shanghai (no. 20ZR1409600). There are no financial disclosures or conflicts of interest for any of the authors. Special thanks to Haidong Xu, Ph.D., who provided special assistance in the hydrophone setup and post-analysis of the audio data.

\section{References}

1 S. C. Joseph, E. Rizk, and R. S. Tubbs: Anatomy, Imaging and Surgery of the Intracranial Dural Venous Sinuses, R. S. Tubbs, Ed. (Elsevier, New York, 2020) pp. 205-220. https://doi.org/10.1016/B978-0-323-65377$\underline{0.00025-8}$

2 D. J. Eisenman: Otol. Neurotol. 32 (2011) 1116. https://doi.org/10.1097/MAO.0b013e31822a1c7d

3 Y. L. Hsieh, X. B. Xu, Y. D. Hsieh, Y. C. Hsieh, D. Wang, P. Ping, and W. Q. Wang: Auris Nasus Larynx 48 (2021) 852. https://doi.org/10.1016/j.anl.2021.01.013

4 A. Sismanis: Otolaryngol. Clin. North. Am. 36 (2003) 389. https://doi.org/10.1097/MAO.0b013e31822alc7d

5 Y. L. Hsieh and W. Q. Wang: Otol. Neurotol. 41 (2020) e132. https://doi.org/10.1097/MAO.0000000000002464

6 P. Raghavan, Y. Serulle, D. Gandhi, R. Morales, K. Quinn, K. Angster, R. Hertzano, and D. Eisenman: Am. J. Neuroradiol. 37 (2016) 136. https://doi.org/10.3174/ajnr.A4511

7 D. J. Eisenman, P. Raghavan, R. Hertzano, and R. Morales: Laryngoscope 128 (2018) S1. https://doi. org/10.1002/lary.27218

8 G. P. Wang, R. Zeng, Z. H. Liu, X. H. Liang, J. F. Xian, Z. C. Wang, and S. S. Gong: Acta Otolaryngol. 134 (2014) 7. https://doi.org/10.3109/00016489.2013.831479

9 K. Bhatnagar, A. T. Lataille, and D. J. Eisenman: Am. J. Otolaryngol. 41 (2020) 102647. https://doi. org/10.1016/j.amjoto.2020.102647

10 Y. L. Hsieh and W. Q. Wang: Sens. Mater. 33 (2021) 2013. https://doi.org/10.18494/SAM.2021.3284

11 P. Guo and W. Q. Wang: Clin. Exp. Otorhinolaryngol. 8 (2015) 111. https://doi.org/10.3342/ceo.2015.8.2.111

12 Y. L. Hsieh, X. B. Xu, P. Guo, and W. Q. Wang: Auris Nasus Larynx 48 (2021) 1181. https://doi.org/10.1016/j. anl.2020.06.012

13 S. Tian, X. Fan, Y. Wang, Z. Liu, and L. Wang: J. Biomech. 84 (2019) 197. https://doi.org/10.1016/j. jbiomech.2018.12.049

14 R. Ewert and W. Schroder: J. Comput. Phys. 188 (2003) 365. https://doi.org/10.1016/S0021-9991(03)00168-2

15 K. Valluru, J. Parkhill, A. Gautam, H. Haraldsson, E. Kao, J. Leach, A. Wright, M. Ballweber, K. Meisel, D. Saloner, and M. Amans: Otol. Neurotol. 41 (2020) e7. https://doi.org/10.1097/MAO.0000000000002452

16 S. N. Park, J. S. Han, J. M. Park, H. J. Jin, H. A. Joo, J. T. Park, J. Y. Koo, Y. Kim, M. Y. Kwak, J. W. Seo, C. I. Song, and H. J. Park: Clin. Otolaryngol. 45 (2020) 280. https://doi.org/10.1111/coa.13493

17 S. Y. Lee, M. K. Kim, Y. J. Bae, G. S. An, K. Lee, B. Y. Choi, J. W. Koo, and J. J Song: Sci. Rep. 10 (2020) 18194. https://doi.org/10.1038/s41598-020-75348-3

18 J. J. Song, G. S. An, I. Choi, D. De Ridder, S. Y. Kim, H. S. Choi, J. H. Park, B. Y. Choi, J. W. Koo, and K. Lee: Otol. Neurotol. 37 (2016) 613. https://doi.org/10.1097/MAO.0000000000001005

19 Y. L. Hsieh, Y. Z. Wu, H. Wang, X. B. Xu, P. Guo, X. Wang, Y. D. Hsieh, H. Y. Lu, and W. Q. Wang: ORL (2021) 1. https://doi.org/10.1159/000517610 


\section{About the Authors}

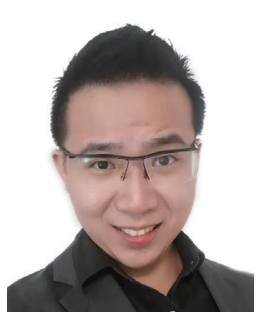

Yue-Lin Hsieh received his M.D. degree in 2019 and completed a three-year residency training program at the Fudan University-affiliated Eye \& ENT Hospital, Department of Otorhinolaryngology-Head and Neck Surgery from 2016 to 2019. He is currently a Ph.D. candidate of Fudan University. He is particularly dedicated to clinical management and biomedical investigation of pulsatile tinnitus. Cerebrospinal fluid/dural venous sinus hemodynamics, endolymphatic hydrops, and middle ear mechanics are also his research interests. (tb22521208@hotmail.com)

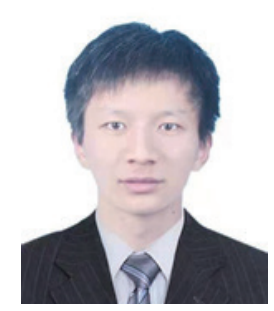

Xing Wang received his postgraduate degree in vehicle engineering from Xiamen University of Technology in 2018. He is an experienced acoustic engineer specializing in computational simulations of fluid-structure interactions. His primary research interests include aero-acoustic and hydrodynamic engineering applications.

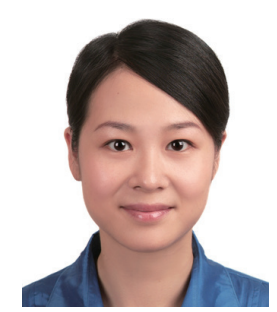

Xiaobing Xu received her Ph.D. degree from the Fudan University-affiliated Eye \& ENT Hospital, Department of Otorhinolaryngology-Head and Neck Surgery, China, in 2007. She is currently an attending doctor at the Department of Otorhinolaryngology-Head and Neck Surgery, and she has been engaged in work on ultrasound since 2013. She is currently dedicated to the Doppler ultrasonographic diagnosis of superficial organs and head and neck blood vessels. (xxb_hy@hotmail.com)

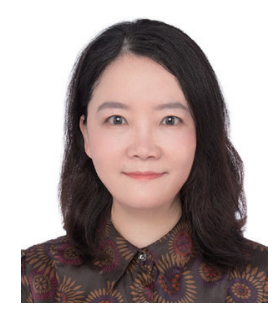

Yongzhen $\mathbf{W u}$ received her Ph.D. degree from the Fudan University-affiliated Eye \& ENT Hospital in 2009. Currently, she is a deputy chief doctor specializing in the medical management of tinnitus and intratympanic injection in the Division of Inner Ear, Department of Otorhinolaryngology-Head and Neck Surgery. She is a member of the audiometric branch of the Department of Otorhinolaryngology-Head and Neck Surgery of Shanghai and China Medical Association. Her primary research interests are audiometric/ psychoacoustic assessments and the treatment of tinnitus and sudden deafness. (wgek2018@vip.163.com)

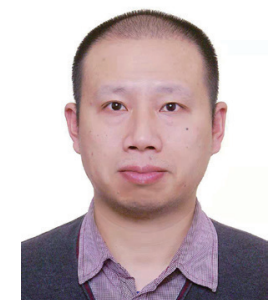

Shenjiang Wang received his bachelor's degree in medical image science from Tongji University in 2007. He has been working for the Department of Radiology of the Fudan University-affiliated Eye and ENT Hospital for nearly 20 years. He is proficient in diagnosis and scanning techniques for computed tomography and magnetic resonance imaging. Resting-state functional magnetic resonance and intravenous gadolinium inner ear magnetic resonance imaging are his research interests. (eent001@sina.com) 


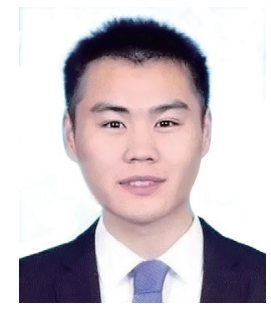

Dengtao Yu received his postgraduate degree in fluid mechanics from University of Shanghai for Science and Technology. Currently, he works as an engineering technician at the Institute of Gas Turbine, Shanghai Electric Gas Turbine Co., Ltd. His research interests involve numerical investigation of aero/fluid dynamics and oscillating airfoils.

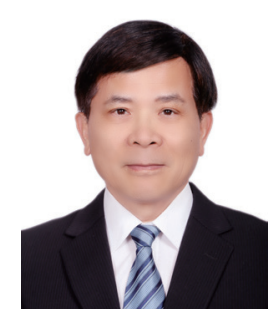

Yi-Chern Hsieh received his Ph.D. degree from Yun-Lin Institute of Technology, ROC, in 2009. He has been engaged in the application of a variety of adaptive scientific computation methods in various engineering problems. He has been a professor of the Department of Power Mechanical Engineering of National Formosa University since 2020. At present, he is focusing on the fields of medical engineering, PET manufacturing, and acoustic engineering.

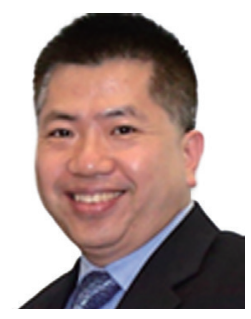

Wuqing Wang received his Ph.D. degree from the Fudan University-affiliated Eye \& ENT Hospital, Department of Otorhinolaryngology-Head and Neck Surgery, China, in 2003. He is a professor of otolaryngology-head and neck surgery and chief of endoscopic ear surgery in the Division of Neurotology and Skull Base Surgery of the Fudan University-affiliated Eye \& ENT Hospital. He is currently a leading otologic surgeon specializing in endoscopic ear surgery in China. He is a member of the Association for Research in Otolaryngology, American Academy of Otolaryngology-Head and Neck Surgery, The International Association of Physicians in Audiology, and the Bárány Society. His current research interests involve surgical management and biomedical investigation of chronic otitis media, Ménière's disease, vestibular migraine, endolymphatic hydrops, idiopathic sudden sensorineural hearing loss, and pulsatile tinnitus. (wwuqing@189.cn, wwuqing@eent.shmu. edu.cn) 